\title{
Prevalence of Dirofilaria immitis Infection in Living Raccoon Dogs Assessed by Hematological Examination
}

\author{
Nobuhide KIDO $^{1,2) *}$, Yuko WADA ${ }^{1)}$, Maya TAKAHASHI ${ }^{1)}$, Chihiro KAMEGAYA ${ }^{1)}$, Tomoko OMIYA ${ }^{1)}$ and \\ Yasuhiko YAMAMOTO ${ }^{1)}$
}

${ }^{1)}$ Kanazawa Zoological Gardens, 5-15-1 Kamariya-higashi, Kanazawa-ku, Yokohama 236-0042 and 2) Nogeyama Zoological Gardens, 63-10 Oimatsu-cho, Nishi-ku, Yokohama 220-0032, Japan

(Received 18 November 2010/Accepted 17 January 2011/Published online in J-STAGE 31 January 2011)

ABSTRACT. The prevalence of Dirofilaria immitis in free-ranging raccoon dogs (Nyctereutes procyonoides viverrinus) was examined in the southeast region of Kanagawa Prefecture, Japan, using a rapid immunomigration (RIM) test kit. Between April 2007 and March 2010, we examined 108 raccoon dogs rescued and housed by the Kanazawa Zoological Garden. D. immitis infection was found in 8 $(7.4 \%)$ raccoon dogs. This is the first report to reveal the prevalence of D. immitis infection in living raccoon dogs. The prevalence of the infection was lower than previously reported values obtained on postmortem examination. One reason might be that the present study included young raccoon dogs infected with immature worms. Significant high-risk areas of D. immitis infection in the raccoon dogs were not observed.

KEY WORDS: Dirofilaria immitis, Nyctereutes procyonoides, prevalence, raccoon dog.

The nematode Dirofilaria immitis infects domestic dogs (Canis familiaris) and cats (Felis catus). Several wild animals are known to be infected with this parasite, including wolves (Canis lupus) [14], free-ranging coyotes (Canis latrans) [4], island foxes (Urocyon littoralis) [11], harbor seal (Phoca vitulina) [3], and black-footed ferrets (Mustela nigripes) [17]. D. immitis has also been detected in other non-carnivore species: free-ranging armadillos (Dasypodidae) [1], pale-headed saki monkeys (Pithecia pithecia) [2], and horse [15]. Furthermore, the nematode causes human pulmonary dirofilariasis [5]. Thus, zoonotic $D$. immitis in animal hosts should be monitored regularly. The raccoon dog (Nyctereutes procyonoides viverrinus), originally distributed throughout Japan, is a member of the Canoidea superfamily [13]; its habitat coincides with the area endemic for D. immitis. Several studies have documented D. immitis infection in the raccoon dog $[6,16]$.

This study aimed at investigating the prevalence of $D$. immitis in living raccoon dogs in the southeast region of Kanagawa Prefecture, Japan, using a commercially available rapid immunomigration (RIM) test kit that detects the presence of circulating antigen mainly originating from the reproductive tract of adult female worms. All previous reports concerning its prevalence were based on postmortem examination $[6,16]$.

The prevalence of $D$. immitis was examined in 108 raccoon dogs rescued due to severe scabies by Kanazawa Zoological Garden between April 2007 and March 2010. Thirteen raccoon dogs were examined from April 2007 to March 2008, 40 from April 2008 to March 2009, and 55 from April 2009 to March 2010. The raccoon dogs were

\footnotetext{
* Correspondence to: Kido, N., Nogeyama Zoological Grandens, 63-10 Oimatsu-cho, Nishi-ku, Yokohama 220-0032, Japan. e-mail: nobukido1977@yahoo.co.jp
}

found in the southeast region of Kanagawa Prefecture, Japan, including the areas Kanazawa Ward, Yokohama $\left(35^{\circ} 34^{\prime} \mathrm{N}, 139^{\circ} 62^{\prime} \mathrm{E}\right)$ (9 animals), Fujisawa $\left(35^{\circ} 20^{\prime} \mathrm{N}\right.$,

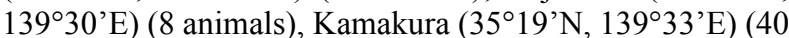
animals), Zushi ( $\left.35^{\circ} 18^{\prime} \mathrm{N}, 139^{\circ} 35^{\prime} \mathrm{E}\right)$ (16 animals), Hayama $\left(35^{\circ} 16^{\prime} \mathrm{N}, 139^{\circ} 35^{\prime} \mathrm{E}\right)$ (22 animals), Yokosuka $\left(35^{\circ} 17^{\prime} \mathrm{N}\right.$, $\left.139^{\circ} 41^{\prime} \mathrm{E}\right)$ (4 animals), and Miura ( $\left.35^{\circ} 08^{\prime} \mathrm{N}, 139^{\circ} 37^{\prime} \mathrm{E}\right)(9$ animals). Raccoon dogs estimated to be $<6$ months of age according to their body length and tooth eruption pattern were eliminated from the sample population.

Blood samples from raccoon dogs were collected from the cephalic vein under manual restraint for hematological and serum biochemical analyses. Ivermectin (Ivomec ${ }^{\circledR}$ Injection, Merial Japan Limited, Tokyo, Japan) was subcutaneously administered to all raccoon dogs for scabies, and a lactated Ringer's solution intravenous fluid (Solulact ${ }^{\mathbb{R}}$, Terumo Corp., Tokyo, Japan) was intravenously given to marasmic raccoon dogs. Samples for the examination of $D$. immitis infection were immediately preserved in EDTA2Na tubes (Venoject ${ }^{\circledR}$ II, Terumo Corp., Tokyo, Japan), and stored at $15-20^{\circ} \mathrm{C}$ until assay. Antigens in the blood samples were examined within the day by the RIM test kit following the manufacturer's instructions. For the microscopic detection of circulating microfilariae, microhematocrit tubes including the samples were centrifuged at 12,000 rpm for $5 \mathrm{~min}$ and then the buffy coats were smeared [10].

To evaluate the accuracy of the RIM test kit designed to detect the presence of the antigen of the adult female $D$. immitis antigen (Vet Assist ${ }^{\circledR}$ DIRO, Arysta LifeScience Corp., Tokyo, Japan), 20 samples were retested using another commercially available immunochromatographic kit (Solo Step ${ }^{\circledR}$ CH, Novartis Animal Health K.K., Tokyo, Japan). In addition, the heart, vena cava, aorta, pulmonary arteries, lungs, and liver were examined for the presence of 
D. immitis at necropsy on 16 animals (12 tested by Vet Assist ${ }^{\circledR}$ DIRO and 4 by the 2 different kit methods) dying to severe asthenia.

The prevalence of $D$. immitis infection is shown in Table 1. The prevalence in each area is as follows: $11.1 \%(1 / 9)$ in Kanazawa Ward, Yokohama; 10.0\% (4/40) in Kamakura; $6.3 \%(1 / 16)$ in Zushi; and 9.1\% (2/22) in Hayama. No raccoon dogs infected with $D$. immitis were found in other areas. Microfilariae were not detected in any of the samples.

The results of the 2 different kit methods were identical. Of the 20 samples assayed with both methods, 2 were positive with Vet Assist ${ }^{\circledR}$ DIRO and Solo Step ${ }^{\circledR} \mathrm{CH}$; remaining 18 samples were negative with both methods. On postmortem examination, 1 positive case detected by Vet Assist ${ }^{\circledR}$ DIRO had 5 immature nematodes. Another positive case detected by the 2 different kit methods had 18 immature nematodes. These were identified microscopically as being $D$. immitis according to the number and position of the papillae on the worm heads and tails. Parasites were not detected in any of the postmortem examinations of the other 14 cases, including the 10 that tested negative by Vet Assist ${ }^{\circledR}$ DIRO and the 4 that tested negative by the 2 different kit methods. The habitats of the raccoon dogs infected with $D$. immitis were plotted on a map (Fig. 1). Chi-square test did not show any significant difference in prevalence by area.

In the present study, the prevalence of $D$. immitis infection in living raccoon dogs was $7.4 \%$ according to the RIM test. Although the kit detects only the antigen of adult female $D$. immitis nematodes, this is the first report to reveal $D$. immitis prevalence in living raccoon dogs. The prevalence is lower than previously reported values $(24.3 \%$, $\mathrm{n}=37 ; 16.0 \%, \mathrm{n}=46$ ) in necropsied raccoon dogs collected in the Kanagawa area by other researchers $[7,16]$. These previous reports cannot be directly compared with the present study because the former findings were collected during postmortem examination. The following reasons for the differences were considered. First, although the raccoon dogs that were estimated to be $<6$ months of age were eliminated from the sample population, the present study might have contained some young raccoon dogs infected with immature worms. The kit method is insensitive to the early stages of infection [12]; therefore, the detected prevalence based on the kit method possibly is lower than that based on postmortem examination. Although the age of raccoon dogs in the present study was not determined, the previous report described that the free-range population of raccoon dogs in Okayama Prefecture in 1983 comprised animals $<3$ years of age [9]. Second, a postmortem examination may have had higher sensitivity than hematological examination for young raccoon dogs because postmortem examination can detect immature worms. On the other hand, earlier reports described that the antigen-based methods had greater sensitivity than earlier studies of heartworm prevalence in canids that could identify adult worms and microfilariae in tissues and blood $[11,12]$. This criterion may be fulfilled in the high-age population, e.g., in domestic dogs. Further studies
Table 1. Prevalence of Dirofilaria immitis infection in raccoon $\operatorname{dog}$ (Nyctereutes procyonoides viverrinus) by hematological examination

\begin{tabular}{lcc}
\hline \multirow{2}{*}{ Examined period } & \multicolumn{2}{c}{ Number of raccoon dog } \\
\cline { 2 - 3 } & examined & positive (\%) \\
\hline April 2007 - March 2008 & 13 & $1(7.7 \%)$ \\
April 2008 - March 2009 & 40 & $3(7.5 \%)$ \\
April 2009 - March 2010 & 55 & $4(7.3 \%)$ \\
\hline Total & 108 & $8(7.4 \%)$ \\
\hline
\end{tabular}

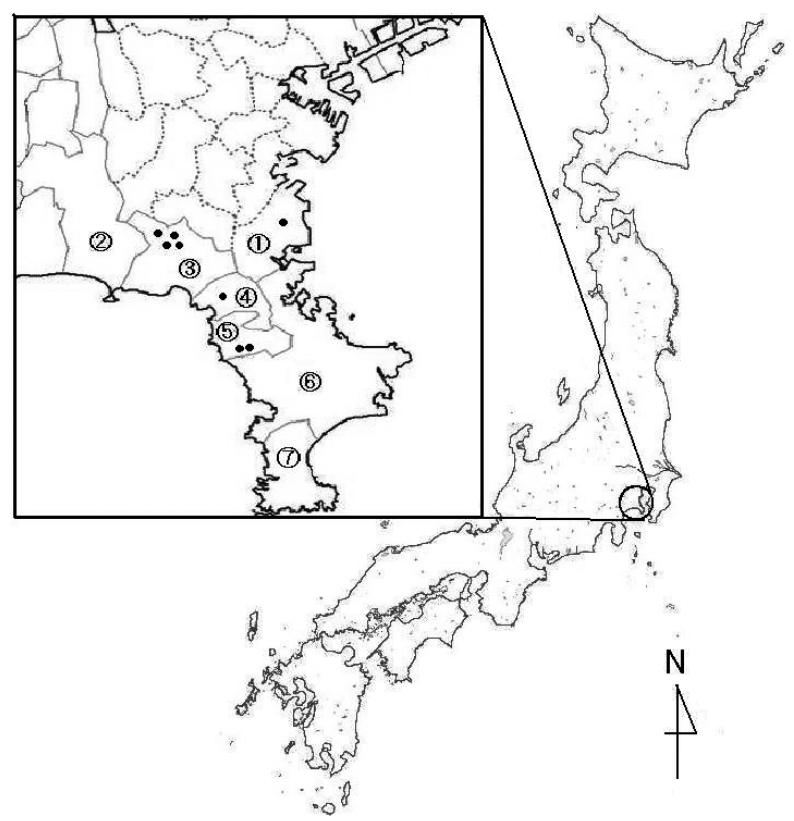

Fig. 1. (): The place where a raccoon dog (Nyctereutes procyonoides viverrinus) infected with Dirofilaria immitis was rescued between April 2007 and March 2010. Study areas: (1) Kanazawa Ward, Yokohama; (2) Fujisawa; (3) Kamakura; (4) Zushi; (5) Hayama; (6) Yokosuka; and (7) Miura.

might be required to reveal the relation between the age of the raccoon dogs and the prevalence of $D$. immitis infections.

Although clinical examinations using antigen-based kits have been restricted to domestic dogs, 20 retested samples using 2 different kits and 16 necropsy cases found the accuracy of Vet Assist ${ }^{\circledR}$ DIRO for diagnosing D. immitis infections in raccoon dogs. In addition, the prevalence of $D$. immitis infection using an antigen-based kit has been reported in black-footed ferrets and island foxes [10, 17].

The prevalence of $D$. immitis infection in living raccoon dogs in the present study is much lower than that reported in domestic dogs (55.0\% in 1983 and $57.5 \%$ in 1984) in the Kanagawa area [6]. To our knowledge, the latest research revealed the prevalence in domestic dogs was $46.8 \%$ in 1997 in the Saitama area [8]. One reason is that the mean age of raccoon dogs in this study may be less than that of the 
domestic dog population. Furthermore, the raccoon dog has been demonstrated to be resistant to $D$. immitis infection. Experimental infection of raccoon dogs by $D$. immitis showed that the average percentage of recovered worms was almost 10 times lower than that in the domestic dog, and their growth was limited [7].

Microfilariae were not identified in the present study. We can attribute 3 reasons for this finding. First, due to the short life span of raccoon dogs, there was insufficient time for $D$. immitis to mature. An earlier report stated that 14 worms in 8 raccoon dog carcasses were mostly immature [6]. Second, microfilaria growth might be restricted in raccoon dogs. In an experimental $D$. immitis infection in raccoon dogs, microfilaremia was observed for 180 days after inoculation but disappeared at around 300 days [7]. Third, the buffy coat examination for $D$. immitis microfilariae is insensitive when blood microfilariae are present at low densities $(50-100 / \mathrm{m} l)$ [12].

Although in the present study significant high-risk areas of $D$. immitis infection were not observed, the study revealed areas of frequent $D$. immitis incidence, particularly the northern part of Kamakura and the southern part of Hayama. Since the number of the raccoon dogs in the present study was small, further studies might be required to reveal whether the prevalence of $D$. immitis is high in specific areas.

\section{REFERENCES}

1. Deem, S. L., Noss, A. J., Fiorello, C. V., Manharth, A. L., Robbins, R. G. and Karesh, W. B. 2009. Health assessment of freeranging three-banded (Tolypeutes matacus) and nine-banded (Dasypus novemcinctus) armadillos in the Gran Chaco, Bolivia. J. Zoo Wildl. Med. 40: 245-256.

2. Gamble, K. C., Fried, J. J. and Rubin, G. J. 1998. Presumptive dirofilariasis in a pale-headed saki monkey (Pithecia pithecia). J. Zoo Wildl. Med. 29: 50-54.

3. Medway, W. and Wieland, T. C. 1975. Dirofilaria immitis infection in a harbor seal. J. Am. Vet. Med. Assoc. 167: 549550 .

4. Miller, D. L., Schrecengost, J., Merrill, A., Kilgo, J., Ray, H. S., Miller K. V. and Baldwin, C. A. 2009. Hematology, parasitology, and serology of free-ranging coyotes (Canis latrans) from South Carolina. J. Wildl. Dis. 45: 863-869.

5. Miyoshi, T., Tsubouchi, H., Iwasaki, A., Shiraishi, T., Nabeshima, K. and Shirakusa, T. 2006. Human pulmonary dirofilariasis: a case report and review of the recent Japanese literature. Respirology 11: 343-347.

6. Nakagaki, K., Suzuki, T., Hayama, S. I. and Kanda, E. 2000. Prevalence of dirofilarial infection in raccoon dogs in Japan. Parasitol. Int. 49: 253-256.

7. Nakagaki, K., Yoshida, M., Nogami, S. and Nakagaki, K. 2007. Experimental infection of Dirofilaria immitis in raccoon dogs. J. Parasitol. 93: 432-434.

8. Nogami, S. and Sato, T. 1997. Prevalence of Dirofilaria immitis infection in cats in Saitama, Japan. J. Vet. Med. Sci. 59: 869-871.

9. Obara, I. 1983. Age structure of Nyctereutes procynoides viverrinus from the central and north regions of Okayama Prefecture. Honyu Doubutsugaku Zashi 9: 204-207 (in Jpanaese).

10. Oishi, I. 1995. Dirofilaria immitis. pp. 110-127. In: Veterinary Clinical Parasitology (Small Animal), 1st ed. (Veterinary Clinical Parasitology Editorial Committee ed.), Buneido Inc., Tokyo (in Japanese).

11. Roemer, G. W., Coonan, T. J., Garcelon, D. K., Starbird, C. H. and McCall, J. W. 2000. Spatial and temporal variation in the seroprevalence of canine heartworm antigen in the island fox. J. Wildl. Dis. 36: 723-728.

12. Rubin, S. B., Nelson, C. T., Carithers, D., Graham, W., Buzhardt, L. F., Jones, S., Levy, J., Stannard, R., RobertsonPlouch, C., Blagburn, B., MacCall, J. W. and Guerrero, J.; Executive Board of the American Heartworm Society. 2010. Diagnosis, Prevention, and Management of Heartworm (Dirofilaria immitis) Infection in Dogs, American Heartworm Society, Delaware.

13. Saeki, M. 2009. Raccoon dog. pp. 216-217. In: The Wild Mammals of Japan, 1st ed. (Ohdachi, S. D., Ishibashi, Y., Iwasa, M. A. and Saitoh, T. eds.), Shoukado Inc., Kyoto.

14. Segovia, J. M., Torres, J., Miquel, J., Llaneza, L. and Feliu, C. 2001. Helminths in the wolf, Canis lupus, from north-western Spain. J. Helminthol. 75: 183-192.

15. Thurman, J. D., Johnson, B. J. and Lichtenfels, J. R. 1984. Dirofilariasis with arteriosclerosis in a horse. J. Am. Vet. Med. Assoc. 185: 532-533.

16. Uchida, A., Uchida, K., Kawakami, Y. and Murata, Y. 1999. Helminthological surveys of raccoon dogs captured in Tokyo and Kanagawa Prefectures. J. Jap. Vet. Med. Assoc. 52: 715721 (in Japanese).

17. Wisely, S. M., Howard, J., Williams, S. A., Bain, O., Santymire, R. M., Bardsley, K. D. and Williams, E. S. 2008. An unidentified filarial species and its impact on fitness in wild populations of the black-footed ferret (Mustela nigripes). $J$. Wildl. Dis. 44: 53-64. 